\title{
Students' conversion from electric field line diagrams to other representations
}

\author{
Esmeralda Campos, \\ Escuela de Ingenieria y Ciencias, Tecnologico de Monterrey, Monterrey, Mexico \\ Genaro Zavala \\ Escuela de Ingenieria y Ciencias, Tecnologico de Monterrey, Monterrey, Mexico \\ Facultad de Ingenieria, Universidad Andres Bello, Santiago, Chile \\ Kristina Zuza and Jenaro Guisasola \\ Department of Applied Physics, University of the Basque Country, San Sebastian, Spain
}

\begin{abstract}
In electricity and magnetism (E\&M) courses, students have difficulties understanding the concept of electric field. Because of its abstract nature, students can only understand the concept of electric field through the use of representations, such as the algebraic notation, the vector field representation and electric field lines diagrams. We aim to analyze how students understand the concept of electric field when converting from electric field lines diagrams to the vector field plot and to the algebraic notation. We conducted a study with 64 introductory engineering physics students after their E\&M course. Students answered a question shown with an electric field lines diagram and were asked to convert to either the vector field plot or the algebraic notation. We analyzed students' answers based on how they recognize the magnitude and direction of the field in the diagram and convert to the desired representations. The results suggest that most students have difficulties interpreting the electric field lines diagram.
\end{abstract}




\section{INTRODUCTION}

In electricity and magnetism (E\&M) courses, students have difficulties understanding the concept of electric field. Comprehending the meaning of the electric field concept is a crucial part of developing a Maxwellian profile of field [1]. Students' difficulties grasping this concept are attributed to their lack of experience dealing with abstract concepts and mathematical formalisms [2]. Because of its abstract nature, students can only understand the concept of electric field through the use of representations [3], such as the algebraic notation, the vector field plot and the electric field line diagram. In this study, we explore how students interpret an electric field line diagram using the theory of semiotic representations as a framework for our analysis. We asked students to convert from an electric field lines diagram to the respective algebraic and vector plot representations. In light of this theory, students' abilities to recognize and convert between representations of the electric field illustrate students' understanding of this concept. In this study, we present the categories that emerged from students' answers and the relevance of our findings.

\section{A. Theory of semiotic representations}

A representation is understood as something that stands for something else [3]. Representations can be physical (e.g. photographs) or semiotic (e.g. verbal descriptions). Mathematical objects are only accessible through semiotic representations, therefore the cognitive activity in the learning of mathematical objects relies on the transformation between semiotic representations [3]. Registers of semiotic representations use the same symbols, rules and associations to represent an object, which allows to make transformations. The possible transformations are treatments (i.e. within the same register) and conversions (i.e. between different registers). According to Duval [3], difficulties in the learning of mathematical concepts can be traced back to the recognition of the same mathematical object in different semiotic representations that usually have nothing in common, and the conversion between those representations. In physics education research, several studies have pointed out the difficulties that students have when transforming between different representational formats, such as graphical and symbolic representations $[4,5,6]$

\section{B. Difficulties understanding electric field lines}

The literature reports on several difficulties that students have interpreting the electric field line representation. When interpreting electric field lines, students attach certain reality to the lines [7], they treat them as entities or tubes that transport charge $[8,9]$. Furthermore, they present some difficulties discriminating between electric field lines, the net electric field at a position and the contribution of electric field by each charge at a point [10]. Some students tend to draw electric field lines for each charge in a system, instead of conceiving electric field lines as a snapshot of the net electric field at any position in space [11].

The literature also reports students' difficulties interpreting the magnitude and direction of the electric field in the electric field lines diagram. Students have difficulties relating the magnitude of the field and the diagram of electric field lines [12], and they fail to interpret the density of lines as the relative magnitude of the electric field [7, 13]. Some students confuse the curvature of the field lines with the direction of the electric field [10]. It has also been found that some features of the diagram may create a blocking effect on the correct application of the superposition principle of electric field $[13,14]$.

\section{Problem statement}

Our objective is to analyze how students recognize the magnitude and direction of the electric field in an electric field line diagram and how they convert to the algebraic notation and to the vector field plot. We aim to link students' abilities of recognition and conversion of electric field line diagrams to their understanding of the concept of electric field. We conducted a study to address these objectives. In section II, we report the data collection process and data analysis strategy. In section III, we report the results of our investigation and discuss relevant insights. Finally, in section IV we conclude the study highlighting some implications in the teaching and learning of the concept of electric field.

\section{METHODOLOGY}

We conducted a study with introductory engineering physics students after their E\&M course in a private Mexican university. Students answered a question shown with an electric field lines diagram and were asked to convert to either the vector field plot or the algebraic notation. We analyzed students' answers based on how they recognized the magnitude and direction of the field in the diagram and converted to the desired representations.

\section{A. Data collection}

We applied two variations of a question randomly to 64 engineering physics students upon completion of their E\&M course. The question that we posed to students shows an electric field line diagram of a non-Coulombic electric field, shown in Fig. 1. A similar question, without the concept of electric field, was studied by [4]. As it is shown, the field has circular symmetry and it decreases with the distance from the center. To avoid distractions, we do not show any sources of field in the diagram. We explicitly state that the electric field shown is non-Coulombic. By the time they complete the introductory E\&M course, our students have had explicit instruction about non-Coulombic electric fields while 
studying Faraday's law. The presentation of the problem is coherent with the contents of the E\&M course.

We asked students to convert from the electric field line diagram (source representation) to either the algebraic notation or to the vector field plot (target representations). Students were asked to explain how their target representation is related to the magnitude and direction of the electric field shown. Each student answered only one variation of the question, therefore half of the students converted from the electric field lines diagram to the algebraic notation, and the other half converted to the vector field plot.

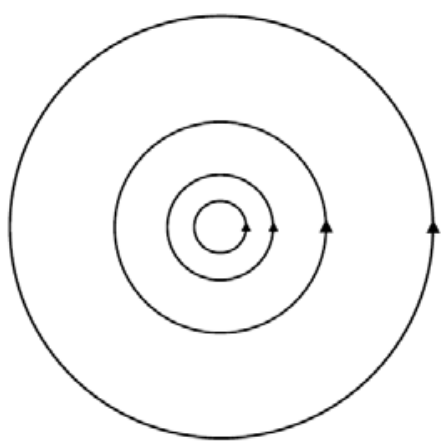

FIG. 1. Electric field line diagram shown to students as a source representation. The electric field shown is non-Coulombic with circular symmetry and it decreases with the distance from the center.

\section{B. Data analysis}

We analyzed data with a qualitative method, considering the abilities of recognition and conversion, and the difficulties that emerged to categorize students' approach to each question. The categorization process was validated by agreement of four researchers, and the classification of students into categories, through Cohen's kappa $(\kappa=0.90)$. Category A means that both the recognition and conversion were successful. In categories B and B', only the recognition or only the conversion was successful respectively. In category $\mathrm{C}$, students made an unsuccessful attempt to recognize and convert. In category $\mathrm{D}$, either students did not attempt to recognize and convert, or their answers were clearly incoherent. Even though the physical situation presented to students is the same, each conversion task requires different processes and meanings. Therefore, we describe the meaning of each category in the specific question. To identify what each category conveys for each question, we considered all drawings, equations and explanations that students answered. We describe and illustrate the explanations that students use to support their answer and the most common difficulties that they have for recognition and conversion. Category D groups students with explanations that differ from any other model with an incoherent structure, and is therefore not analyzed in this contribution. Finally, we summarize the results in Table I, presenting the number of students that were classified in each category, and the percentage in parentheses.

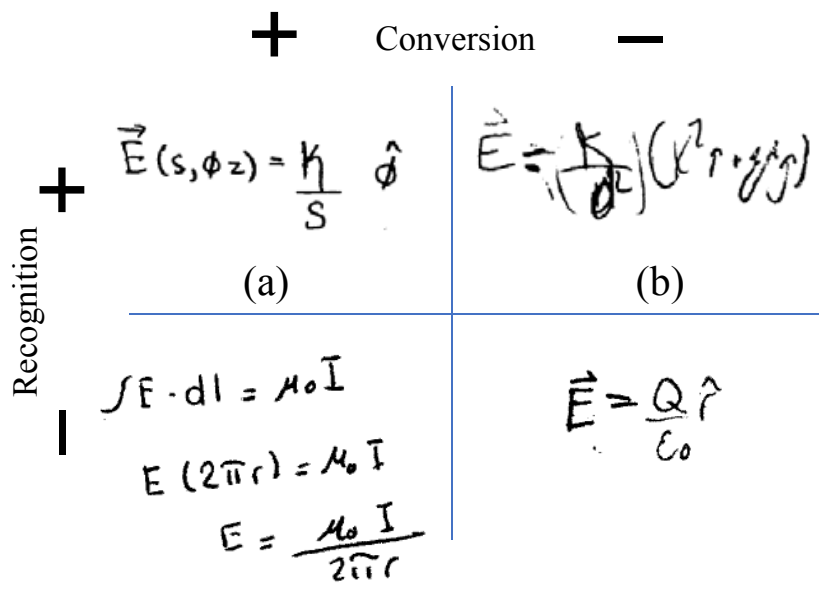

(c)

FIG. 2. Students' recognition and conversion from electric field lines to algebraic expressions. Fig. 2 (a) shows an expression that implies successful recognition and conversion. Fig. 2 (b) shows an example of good recognition, but insufficient conversion of the direction. Fig. 3 (c) shows an inappropriate recognition, relating the electric field to a magnetic source. Fig. 4 (d) shows poor recognition and conversion, relating the electric field to an electric source.

\section{RESULTS AND DISCUSSION}

In this analysis we first present the categories that emerged in the conversion from electric field lines to the algebraic notation. Then, we present the emerging categories in the conversion to the vector field plot. Finally, we present a summary of the results, highlighting the main findings and discuss their implications.

\section{A. Difficulties of recognition and conversion from electric field lines diagram to algebraic notation}

When converting from the electric field lines diagram to the algebraic notation, a successful recognition of the characteristics of the field in the representation is considered in categories $\mathrm{A}$ and $\mathrm{B}$. The difficulties of recognition are considered in categories B' and C. A successful conversion is shown in categories A and B', while an unsuccessful conversion is classified as $\mathrm{B}$ or $\mathrm{C}$.

In category $\mathrm{A}$, students describe that the electric field decreases with the distance from the center and note that the direction is counter-clockwise. The students in this category create an algebraic expression that indicates proportionality and an inverse dependence on the radius, as well as a counter-clockwise direction. For example, one student gave the expression shown in Fig. 2 (a) and explained that "the expression describes the direction because they [the field lines] go around. The magnitude decays as they move away 
from the origin, given that the field lines are further apart" (Student I1-5).

In category $\mathrm{B}$, students make a correct interpretation of the diagram, but their equation does not properly describe the magnitude or the direction of the field. For example, in Fig. 2 (b), the student fails to express the direction of the field in polar coordinates, but states that: "the magnitude decreases with the distance and the direction is circular" (Student I1-21), which implies a good recognition, but a difficulty of conversion.

The difficulties of recognition emerged when students tried to associate the electric field lines diagram to electric or magnetic sources. Fig. 2 (c) shows an example of category B', where students would associate this electric field to a magnetic source. The student explained: "the electric field keeps the same direction as the circle, defined by radius $r$. If the circle is bigger, the magnitude of the field is smaller" (Student I1-27). While the form of the expression is correct, at least in magnitude, the recognition is not successful because, instead of referring to the characteristics of the representation, it seems that they draw from memoristic resources, like the magnetic field produced by a line of current. In category $\mathrm{C}$, students would try to associate the electric field to an electric source, as shown in Fig. 2 (d). Their conversions included $\mathrm{q}$ and/or $\varepsilon_{0}$ as proportionality constants and/or indicated an inverse dependence on $\mathrm{r}^{2}$. We can think that they answered this from a memoristic approach in both categories because in the electric field line diagram shown (Fig. 1) we did not include the source, nor any other constants or parameters of the physical situation.

\section{B. Difficulties of recognition and conversion from electric field lines to vector diagram}

When students convert from electric field lines to the vector field plot, we only found categories $\mathrm{A}$ and $\mathrm{C}$. Categories B and B' did not emerge in students' answers while doing this conversion. Category A includes those students who interpreted that the electric field decreases radially and that the direction is counter-clockwise, tangent to the electric field lines at any point. These students drew enough vectors to represent the changes in magnitude and direction. We show an example in Fig. 3 (a). As it can be seen, this student drew vectors with decreasing length and with the direction tangent to the electric field lines at enough positions, demonstrating appreciation of the changes both in magnitude and direction. In their explanation, the student states: "The vectors are tangent to the field lines, and their magnitude is bigger where field lines are closer (density)" (Student I2-18).

In category $\mathrm{C}$, students made an unsuccessful recognition because they would interpret that the electric field increased radially and draw vectors accordingly. Figure 3 (b) shows an example of a student who correctly interpreted that the direction is tangent to the field lines, but incorrectly interpreted that the magnitude of the field increases radially, and drew vectors accordingly. Also, in their drawing, the vectors touch the field lines at the middle of the arrow, instead of at the starting point. In their explanation, the student stated "the electric field increases in magnitude as you move away from the center of the circle. The direction changes as you move around the circle" (Student I2-29).

This difficulty could mean that students associate the size of the circles with the intensity of the field, instead of recurring to the density of field lines. An ever increasing electric field would mean that its intensity tends to infinity with the radius, which is an impossible physical situation. From these results, we wonder if certain characteristics of the representation might inhibit the meaning making of the physical situation.

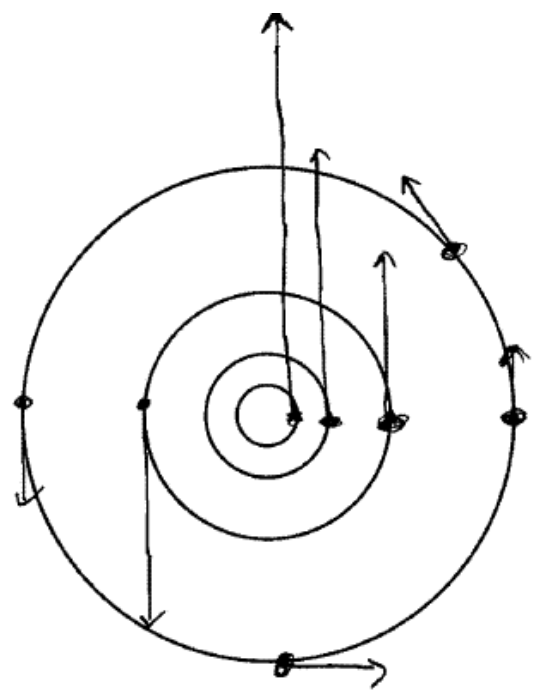

(a)

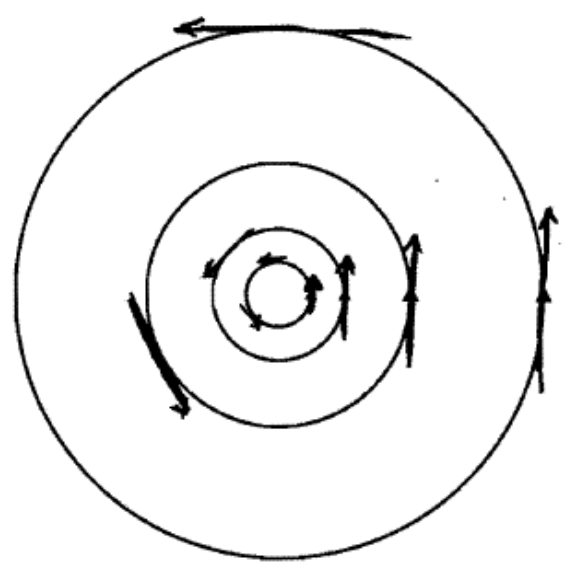

(b)

FIG. 3. Students' recognition and conversion from electric field lines to vector field plots. Fig. 2 (a) shows a vector field plot with the arrows tangent to the electric field lines and decreasing in length. Fig. 2 (b) shows a vector field plot with arrows increasing in length and a few details on the way vectors are drawn. 
TABLE I. Number (and percentage) of students classified in each category when converting from electric field lines to the algebraic notation and the vector field plot.

\begin{tabular}{lcc}
\hline \hline Category & $\begin{array}{c}\text { Electric field lines to } \\
\text { Algebraic notation }\end{array}$ & $\begin{array}{c}\text { Electric field lines to } \\
\text { Vector field plot }\end{array}$ \\
\hline A & $5(15.6)$ & $9(28.1)$ \\
B & $2(6.3)$ & $0(0.0)$ \\
B' & $1(3.1)$ & $0(0.0)$ \\
C & $20(62.5)$ & $21(65.6)$ \\
D & $4(12.5)$ & $2(6.3)$ \\
Total & $32(100.0)$ & $32(100.0)$ \\
B'+C & $21(65.6)$ & $21(65.6)$ \\
\hline \hline
\end{tabular}

\section{Findings and discussion}

We summarize the results of the two questions in Table I. We include the four main categories (A, B, B' and C), the category of incoherent answers (D) and the total number of students who answered the question. Additionally, we highlight in the last row, the sum of students who had difficulties of recognition $\left(B^{\prime}+C\right)$. Our results suggest that students who successfully recognize the characteristics of the electric field in the field lines diagram are able to convert efficiently to the vector field plot and with few difficulties to the algebraic notation. However, the majority of students have problems recognizing the characteristics of the field in the electric field lines diagram, those considered in categories B' and C (65.6\% in both questions).

We found that to convert from the electric field lines representation to the algebraic notation, students tried to associate the field lines diagram with a source, either electric or magnetic, instead of interpreting the characteristics of the field from the information given by the representation. This solving strategy is similar to the behavior observed by Bollen, Van Kampen, Baily, Kelly and De Cock [4] when they gave the students a similar question, without context, and students would answer with the magnitude of the Coulomb force or the electric field created by a point charge. Also, Campos and Zavala [15] reported that electricity and magnetism professors interpret an electric field lines diagram by attempting to identify the sources of field.

When converting from the electric field lines diagram to the vector field plot, we found that students had difficulties relating the density of field lines to the magnitude of the electric field. The most common difficulty of recognition was that students drew vectors that increased in length when farther away from the center. This behavior is consistent with the difficulties found by [4]. Some students would also have the difficulties of conversion highlighted by [4], such as drawing only one set of vectors and the arrows centered at their location. With these findings, we can see how some difficulties that were found previously without context persist within the context of electric field.

In our study, students were asked to convert from the electric field lines directly to the algebraic notation. This lead to a difference in the results obtained, as compared to those reported by [4]. In their study, students first converted to the vector field plot and then to the algebraic notation. They found that students who had already interpreted that the magnitude of the field increases with the distance from the center, would create a mathematical expression that matched their vector field plot. We did not see this behavior in our results; rather, our students would more consistently try to identify the sources of field. This is an important finding because, it implies that the difficulties that students have converting from one representation to another are carried through different conversion processes. We found that the difficulty of interpreting the increasing magnitude of the field was exclusive of the conversion from the electric field lines to the vector field plot in this problem in particular. This clarifies that the source of this difficulty is created by the conversion task [3], and it leads students to an incomplete understanding of the concept of electric field.

\section{CONCLUSION}

In this study, we analyzed how students recognize the characteristics of the electric field in the electric field line representation, and how they convert to the algebraic notation and vector field plot. We found that students have several difficulties recognizing the characteristics of the electric field in the field line representation. Mainly, when converting to the vector field plot, students are not aware of the density of field lines, and when converting to the algebraic notation, they attempt to associate it with the sources of field.

Our findings have some implications for the teaching and learning of the concept of electric field at the introductory level. We consider that it is important to acknowledge that when students have difficulties interpreting a representation, they carry these difficulties to other representations of the same concept. To have good conceptual understanding, it is necessary to have good synergy between the representations of the concept. When teaching the concept of electric field, we should be aware of the difficulties that may arise from some conversion tasks, to address them during instruction.

This study is limited by the nature of the problem that we posed to students. We only studied how students interpret an electric field lines diagram, but we did not explore how they construct electric field lines diagrams from other representations. More research needs to be done on the recognition and conversion of the electric field in several representations.

\section{ACKNOWLEDGMENTS}

We thank Dr. J. I. Barragues for his contributions to our discussions about Duval's theory of semiotic representations. We also acknowledge the partial financial support of the School of Engineering and Sciences of Tecnologico de Monterrey, the Basque Government Project PIBA IT1349-19 and of the Spanish Government MINECO EDU2015-65359-P 
[1] C. Furió and J. Guisasola, Difficulties in learning the concept of electric field, Sci. Educ. 82, 4 (1998).

[2] R. W. Chabay and B. A. Sherwood, Restructuring the introductory electricity and magnetism course, Am. J. Phys. 74(4), 329-336 (2006).

[3] R. Duval, A cognitive analysis of problems of comprehension in a learning of mathematics, Educ. Stud. Math. 61 (2006).

[4] L. Bollen, P. van Kampen, C. Baily, M. Kelly and M. De Cock, Student difficulties regarding symbolic and graphical representations of vector fields, Phys. Rev. Phys. Educ. Res. 13, 020109 (2017).

[5] D. Nguyen and N. Rebello, Students' Difficulties in Transfer of Problem Solving Across Representations, AIP Conf. Proc. 1179(1), 221 (2009).

[6] M. De Cock, Representation use and strategy choice in physics problem solving, Phys. Rev. ST Phys. Educ. Res. 8, 020117 (2012).

[7] S. Törnkvist, K. A. Pettersson and G. Tranströmer, Confusion by representation: On student's comprehension of the electric field concept, Am. J. Phys. 61, 4 (1993).

[8] Y. Cao and B. M. Brizuela, High school students' representations and understandings of electric fields, Phys. Rev. Phys. Educ. Res. 12, 020102 (2016).
[9] M. C. Pocoví, The effects of a history $\square$ based instructional material on the students' understanding of field lines, J. Res. Sci. Teach. 44, 1 (2007).

[10] J. Li and C. Singh, Investigating and improving introductory physics students' understanding of the electric field and superposition principle, Eur. J. Phys. 38, 055702 (2017).

[11] E. Campos, G. Zavala, K. Zuza and J. Guisasola, Electric field lines: The implications of students' interpretation on their understanding of the concept of electric field and of the superposition principle, Am. J. Phys., 87(8), 660 (2019); https://doi.org/10.1119/1.5100588

[12] M. Saarelainen, A. Laaksonen and P.E. Hirvonen, Students' initial knowledge of electric and magnetic fields - more profound explanations and reasoning models for undesired conceptions, Eur. J. Phys. 28 (2007).

[13] E. Campos and G. Zavala, A Look into Students' Interpretation of Electric Field Lines, in Handbook of Research on Driving STEM Learning with Educational Technologies, edited by M. S. Ramírez-Montoya (pp. 342-364) (IGI Global, 2017).

[14] A. Garza and G. Zavala, Contrasting students' understanding of electric field and electric force, AIP Conf. Proc. 1513, 142 (2013).

[15] E. Campos, and G. Zavala, Exploring instructors' interpretation of electric field lines. Paper presented at Physics Education Research Conference 2017, Cincinnati, OH (2017). 